\title{
Evaluation of growth changes induced by functional appliances in children with Class 11 malocclusion: Superimposition of lateral cephalograms on stable structures
}

\author{
Eunhye $\mathrm{Oh}^{\mathrm{a}}$ (1) \\ Sug-Joon Ahn ${ }^{b}$ \\ Liselotte Sonnesen ${ }^{\mathrm{a}}$ (1)
}

${ }^{a}$ Section of Orthodontics, Department of Odontology, Faculty of Health and Medical Sciences, University of Copenhagen, Copenhagen, Denmark

${ }^{b}$ Department of Orthodontics, School of Dentistry, Seoul National University, Seoul, Korea
Objective: To compare short- and long-term dentoalveolar, skeletal, and rotational changes evaluated by Björk's structural method of superimposition between children with Class 11 malocclusion treated by functional appliances and untreated matched controls. Methods: Seventy-nine prepubertal or pubertal children (mean age, $11.57 \pm 1.40$ years) with Class 11 malocclusion were included. Thirty-four children were treated using an activator with a high-pull headgear (Z-activator), while 28 were treated using an activator without a headgear (E-activator). Seventeen untreated children were included as controls. Lateral cephalograms were obtained before treatment (T1), after functional appliance treatment (T2), and after retention in the postpubertal phase (T3). Changes from T1 to T2 and T1 to T3 were compared between the treated groups and control group using multiple linear regression analysis. Results: Relative to the findings in the control group at T2, the sagittal jaw relationship (subspinalenasion-pogonion, $p<0.001$ ), maxillary prognathism (sella-nasion-subspinale, $p<0.05)$, and condylar growth $(p<0.001)$ exhibited significant improvements in the Z- and E-activator groups, which also showed a significantly increased maxillary incisor retraction $(p<0.001)$ and decreased overjet $(p<0.001)$. Only the E-activator group exhibited significant backward rotation of the maxilla at T2 $(p<0.01)$. The improvements in the sagittal jaw relationship $(p<0.01)$ and dental relationship $(p<0.001)$ remained significant at T3. Condylar growth and jaw rotations were not significant at T3. Conclusions: Functional appliance treatment in children with Class 11 malocclusion can significantly improve the sagittal jaw relationship and dental relationships in the long term.

[Korean J Orthod 2020;50(3):170-180]

Key words: Class 11 malocclusion, Functional appliance, Growth evaluation, Superimposition

Received September 3, 2019; Revised December 13, 2019; Accepted December 13, 2019.

Corresponding author: Liselotte Sonnesen.

Professor, Section of Orthodontics, Department of Odontology, Faculty of Health and Medical Sciences, University of Copenhagen, 20 Nørre Alle, DK-2200 Copenhagen N, Denmark. Tel +45-35-32-66-70 e-mail alson@sund.ku.dk

How to cite this article: Oh E, Ahn SJ, Sonnesen L. Evaluation of growth changes induced by functional appliances in children with Class 11 malocclusion: Superimposition of lateral cephalograms on stable structures. Korean J Orthod 2020;50:170-180.

(C) 2020 The Korean Association of Orthodontists.

This is an Open Access article distributed under the terms of the Creative Commons Attribution Non-Commercial License (http://creativecommons.org/licenses/by-nc/4.0) which permits unrestricted non-commercial use, distribution, and reproduction in any medium, provided the original work is properly cited. 


\section{INTRODUCTION}

Functional appliances have been widely used for growth adaptation/modification treatment in growing children with Class 11 malocclusion, and their effects have been extensively discussed. ${ }^{1-5}$ Some authors documented that functional appliances are effective in improving dentoalveolar and skeletal relationships, while others presented that skeletal effects are minimal relative to those in untreated controls on a long-term basis. ${ }^{1-5}$

In most studies, growth changes induced by functional appliances were conventionally measured on lateral cephalograms, with the use of points and lines for identifying sagittal jaw changes measured on the chin point and vertical changes measured on the mandibular line in relation to the nasion-sella line (NSL). ${ }^{2-5}$ However, it was found that these methods underestimate the vertical displacement and rotations of the jaws, and that the dynamics of jaw rotations are often masked because of instability of the points and lines during growth. ${ }^{6-9}$

Moreover, in many studies, initial correction using functional appliance treatment involved not only forward positioning of the mandible but also vertical opening of the bite and an increase in the lower anterior facial height..$^{4-6}$ Advancement of the chin point at the pogonion may not be evident if the vertical dimension is increased along with the mandibular length. This means that jaw rotations could influence the measurement of sagittal changes.

Björk performed longitudinal implant studies using a series of lateral cephalograms and documented true rotations and growth of the jaws. ${ }^{7-9}$ These studies found relatively stable structures in the anterior cranial base, maxilla and mandible and suggested a technique involving superimposition on the stable structures for the evaluation of true growth changes. ${ }^{7-9}$ However, to the best of our knowledge, no study has reported the true rotational changes in the maxilla and mandible induced by functional appliances with the use of the structural method. ${ }^{4-9}$

Under the assumption that functional appliances affect not only sagittal but also vertical and rotational changes in growing children with Class 11 malocclusion, we compared short- and long-term dentoalveolar, skeletal, and rotational changes evaluated by Björk's structural method of superimposition between children with Class 11 malocclusion treated by functional appliances and untreated matched controls. The null hypothesis was that there is no difference in the changes induced by functional appliances and those that occur without treatment in growing children with Class 11 malocclusion.

\section{MATERIALS AND METHODS}

This study was approved by the Danish Data Protection Agency (no. 2015-57-0121) and the ethical committee of Seoul National University Dental Hospital, Korea (IRB 207/08-16).

\section{Subjects}

A total of 79 prepubertal or pubertal children (40 boys and 39 girls; mean age, $11.57 \pm 1.40$ years) with Class 11 malocclusion were included in this study. Among these children, 34 (19 boys and 15 girls; mean age, $11.82 \pm$ 1.48 years) were treated with a high-pull headgear combined with a Teuscher activator (Z-activator), ${ }^{10}$ while 28 (12 boys and 16 girls; mean age, $11.17 \pm 1.20$ years) were treated with a modified Andresen activator, also known as an Ergenzinger activator (E-activator; Figure 1)," between 2008 and 2015 at the Section of Orthodontics, Department of Odontology, Copenhagen University, Denmark and the Department of Orthodontics, Seoul National University Dental Hospital, Korea. The remaining 17 children ( 9 boys and 8 girls) were included as controls; their data were retrieved from the archives of the classic implant studies by Björk. ${ }^{7-9}$ The craniofacial characteristics and maturation stages of children in
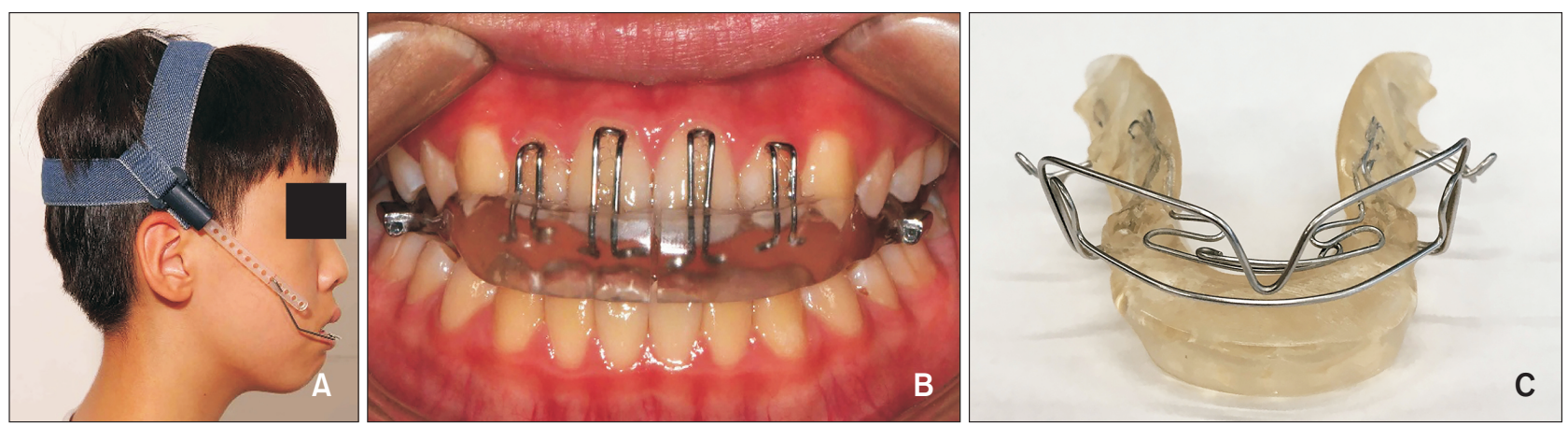

Figure 1. Functional appliances used in the present study. A and B, Z-activator (Teuscher activator with a high-pull headgear) ${ }^{10}$. C, E-activator (modified Andresen activator, also known as an Ergenzinger activator). ${ }^{11}$ 
the control group were matched to those of children in the treated groups in accordance with the inclusion criteria below.

The inclusion criteria were as follows: 1) no previous orthodontic treatment; 2) availability of lateral cephalograms obtained before treatment (T1), after functional appliance treatment (T2), and after more than 1 year of retention, with or without subsequent fixed appliance treatment (T3); 3) subspinale-nasion-supramentale > $4.5^{\circ}$; 4) overjet $>5 \mathrm{~mm}$; 5) pubertal $/$ prepubertal growth peak (cervical stage [CS] 1-4) at $\mathrm{T} 1 ;^{12}$ and 6) postpubertal growth peak (CS 5 and 6 ) at T3. ${ }^{12}$

The exclusion criteria were as follows: 1) fixed appliance treatment with extractions, Class 11 elastics, or other Class II functional devices after functional appliance treatment and 2) the presence of craniofacial syndromes or general diseases.

The power calculation was performed using variables such as condylar growth and sagittal jaw changes from previous studies. ${ }^{1,2}$ For sufficient power (80\%) to identify relevant differences at a 5\% level of significance, and considering an allocation ratio of $1: 1.5$, at least 16 and 24 children were required in the untreated control and each treatment group, respectively.

The Z-activator consists of an activator combined with a high-pull headgear (force vector between the center of resistance of the nasomaxillary complex and maxillary dentition). The appliance was fabricated and adjusted according to the method of Teuscher. ${ }^{10}$ The E-activator is a modified Andresen activator. It is equipped with an additional high labial bow and palatal spring in the maxilla for preventing maxillary incisor tipping along with clasps on all first molars for preventing migration (Figure 1). ${ }^{11}$

A construction bite was recorded under an anterior edge-to-edge relationship with an opening of approximately $2 \mathrm{~mm}$ between the central incisors. In children with excessive overjet, two-step activation was performed. The children in both groups were instructed to wear the appliance daily for 14 hours in the evening and night. The appliances as well as patient compliance were checked every 8 weeks until a Class 1 molar relationship or an appropriate overjet was achieved. If compliance was sufficient, the overjet was found to have decreased by at least $1 \mathrm{~mm}$ at every visit. Children with inadequate compliance and those who required further treatment with other functional appliances were excluded. After the active treatment phase, the children were regularly recalled until the end of the growth peak. If required, fixed appliance treatment was performed during that period.

\section{Methods}

Lateral cephalograms were acquired in centric occlu- sion and the standard mirror position at $\mathrm{T} 1, \mathrm{~T} 2$, and T3. ${ }^{13}$

For the Danish children, lateral cephalograms were acquired using the Philips MEDIO 30 CP X-ray tube (Philips, Eindhoven, Netherlands) with a film-to-focus distance of $180 \mathrm{~cm}$ and a film-to-median plane distance of 10 $\mathrm{cm}$. The constant linear enlargement was $5.6 \%$. For the Korean patients, lateral cephalograms were acquired using the Asahi CX-90 SP device (Toshiba, Tokyo, Japan) with a film-to-focus distance of $150 \mathrm{~cm}$ and a film-tomedian plane distance of $15 \mathrm{~cm}$. The constant linear enlargement was $10 \%$. Correction for the constant linear enlargement was implemented in both groups.

The craniofacial characteristics and growth changes from $\mathrm{T} 1$ to $\mathrm{T} 2$ and $\mathrm{T} 1$ to $\mathrm{T} 3$ were digitally analyzed using TIOPS 2005 ver. 2.12.4 (Total Interactive Orthodontics Planning System [TIOPS], Copenhagen, Denmark; Figures $2-5)^{7-9,14}$ The series of lateral cephalograms were superimposed on the stable structures as per Björk's structural method. ${ }^{7-9}$ Stable anatomical structures in the anterior cranial base were used as reference structures for analysis of vertical and sagittal rotational changes in the jaws. The NSL at baseline was transferred to the subsequent lateral cephalograms as a reference line ( $\mathrm{NSL}^{\text {Ref }}$; Figure 3). The maxilla and mandible were once again superimposed separately on the stable anatomical structures in the respective jaws (T1-T2, $\mathrm{T} 1-\mathrm{T} 3)$, and a reference line was marked on the maxilla (Maxilla ${ }^{\text {Ref }}$ ) and

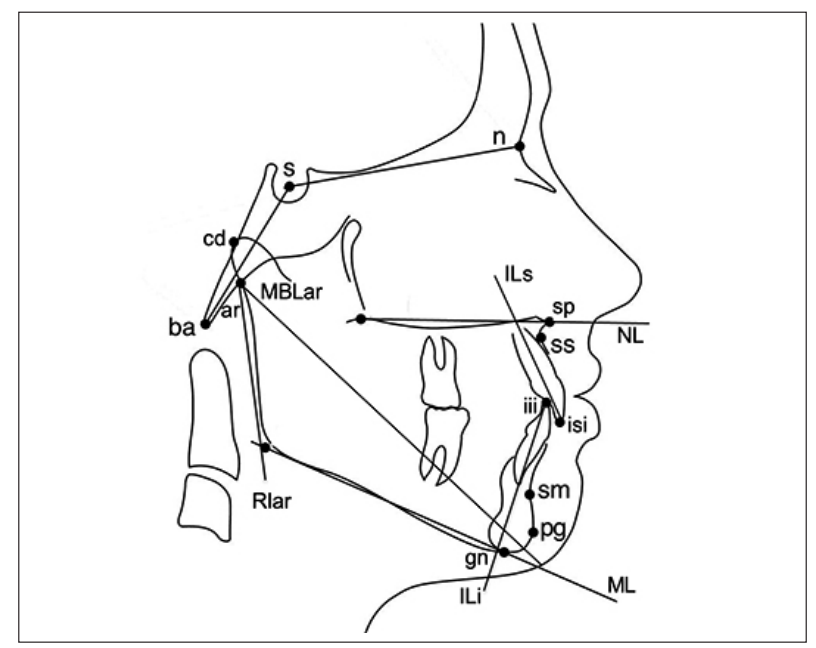

Figure 2. Cephalometric landmarks used for measurements. $^{14}$

$n$, Nasion; s, sella; cd, condylion; ba, basion; ar, articulare; gn, gnathion; pg, pogonion; sm, supramentale; isi, incisal superior incisor; iii, incisal inferior incisor; ss, subspinale; sp, spinale; MBLar, mandibular base line; Rlar, ramus line; ILi, incisal line inferior; ILs, incisal line superior; ML, mandibular line; $\mathrm{NL}$, nasal line. 


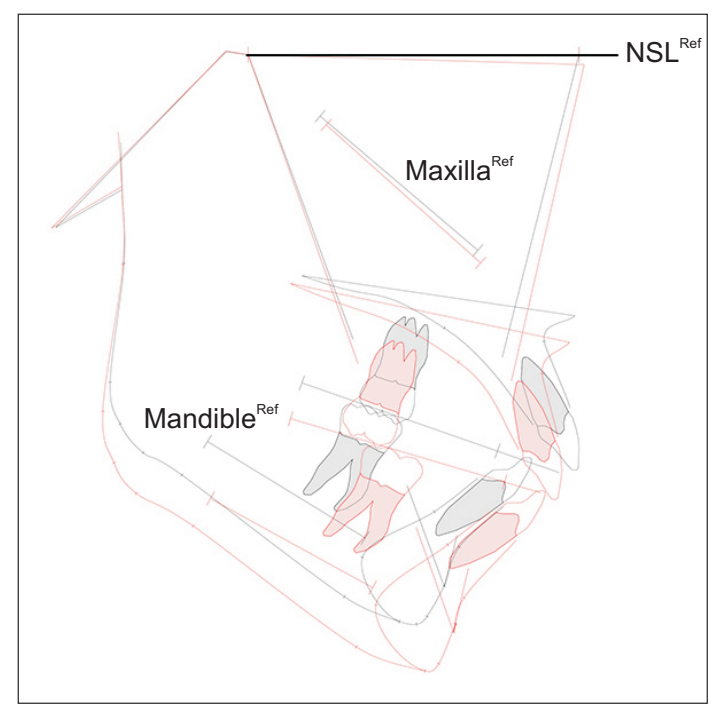

Figure 3. Illustration of superimposition on the stable structures in the anterior cranial base between two stages. ${ }^{9}$

$\mathrm{NSL}^{\text {Ref }}$, Nasion-sella line at before treatment (T1) transferred to the following lateral cephalogram as a reference line when the two sets are superimposed on the stable structures in the anterior cranial base; Maxilla ${ }^{\text {Ref }}$, a reference line drawn in the maxilla when the two sets are superimposed on the stable structures in the maxilla; Mandible ${ }^{\text {Ref }}$, a reference line drawn in the mandible when the two sets are superimposed on the stable structures in the mandible.

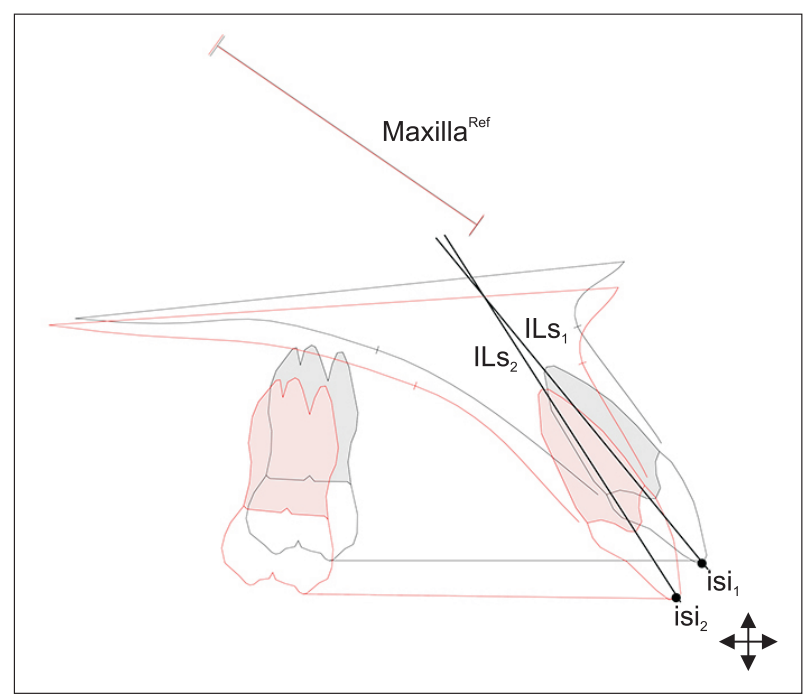

Figure 4. Illustration of superimposition on the stable structures in the maxilla between two stages. ${ }^{9}$

Maxilla ${ }^{\text {Ref }}$, Maxillary reference line; ILs, incisal line superior stage $1 ; I^{\prime} S_{2}$, incisal line superior stage $2 ;$ isi $i_{1}$, incisal superior stage $1 ;$ isi $i_{2}$ incisal superior stage 2 .

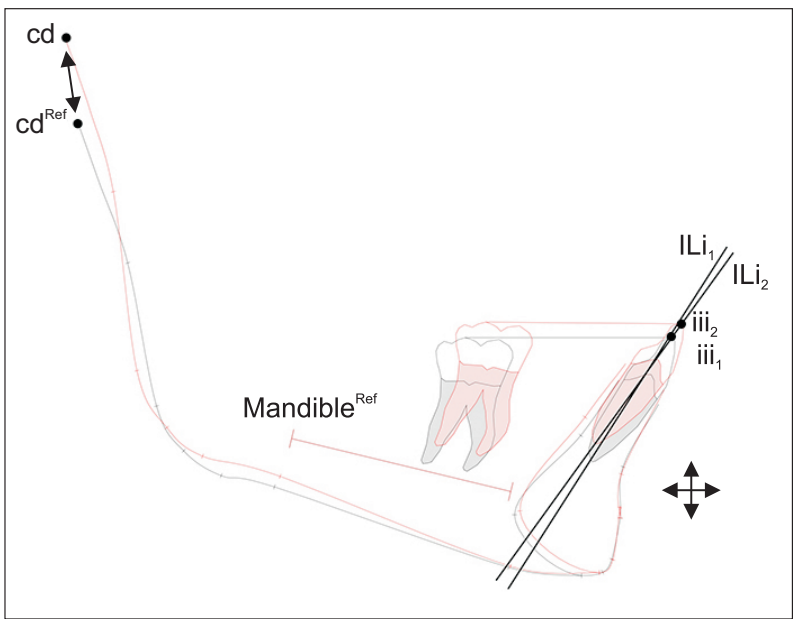

Figure 5. Illustration of superimposition on the stable structures in the mandible between two stages. ${ }^{9}$ $\mathrm{cd}$, Condylion; $\mathrm{cd}^{\mathrm{Ref}}$, condylion at baseline (T1); Mandible ${ }^{\text {Ref }}$, mandibular reference line; $\mathrm{Li}_{1}$, incisal line inferior stage $1 ; \mathrm{ILi}_{2}$, incisal line inferior stage $2 ; \mathrm{iii}_{1}$, incisal inferior stage $1 ; \mathrm{iii}_{2}$, incisal inferior stage 2 .

mandible (Mandible $e^{\text {Ref }}$; Figures 4 and 5)..$^{7-9}$ The angles between $\mathrm{NSL}^{\mathrm{Ref}}$ and Maxilla ${ }^{\mathrm{Ref}}$ and between NSL ${ }^{\mathrm{Ref}}$ and Mandible ${ }^{\text {Ref }}$ were measured for assessing the rotations of the maxilla and mandible, respectively (Figure 3). The dentoalveolar changes and condylar growth were analyzed by superimposition on the stable structures in the maxilla and the mandible..$^{7-9}$ Condylar growth was measured as the linear distance from the condylion at $\left.\mathrm{T} 1 \mathrm{~cd}^{\mathrm{Ref}}\right)$ to the condylion at T2 or T3 when the two sets of lateral cephalograms were superimposed on the stable structures in the mandible. Changes in the cranial base, overjet, overbite, and lower anterior facial height were measured using conventional methods (Figures 2-5, Tables 1-3).

The upper spine morphology and cervical vertebral maturation (CVM) were visually assessed on the lateral cephalograms, ${ }^{12,15}$ and the atlas dimension was digitally measured using TIOPS 2005 (TIOPS; Table 1). ${ }^{16}$ The children were also categorized into two groups according to the treatment timing. Children treated with functional appliances before the pubertal growth peak were included in an early treatment group, whereas those treated during the growth peak were included in a late treatment group. The pubertal growth peak occurs between cervical stage 3 (CS3) and cervical stage 4 (CS4); ${ }^{12}$ therefore, CVM stages that were between cervical stage 1 and CS3 and between CS4 and cervical stage 5 at T2 were considered eligible for the early and late treatment groups, respectively. 
Table 1. Comparison of baseline craniofacial characteristics (T1) between children treated with Class II functional appliances and untreated children

\begin{tabular}{|c|c|c|c|c|c|}
\hline \multirow{2}{*}{ Variable } & \multirow{2}{*}{$\begin{array}{c}\text { Z-activator } \\
\text { (15 girls, } 19 \text { boys) }\end{array}$} & \multirow{2}{*}{$\begin{array}{c}\text { E-activator } \\
\text { (16 girls, } 12 \text { boys) }\end{array}$} & \multirow{2}{*}{$\begin{array}{c}\text { Control } \\
\text { (8 girls, } 9 \text { boys) }\end{array}$} & \multicolumn{2}{|c|}{$p$-value } \\
\hline & & & & Z vs. C & E vs. C \\
\hline CVM methods & & & & NS & NS \\
\hline CS1 & $5(14.7)$ & $7(25.0)$ & $1(5.9)$ & & \\
\hline CS2 & $13(38.2)$ & $11(39.3)$ & $7(41.2)$ & & \\
\hline CS3 & $10(29.4)$ & $7(25.0)$ & $9(52.9)$ & & \\
\hline CS4 & $6(17.6)$ & $3(10.7)$ & $0(0)$ & & \\
\hline Treatment timing & & & & NS & NS \\
\hline Early (pre-peak) & $17(50.0)$ & $17(60.7)$ & $8(47.1)$ & & \\
\hline Late (on-peak) & $17(50.0)$ & $11(39.3)$ & $9(52.9)$ & & \\
\hline \multicolumn{6}{|l|}{ Upper spine morphology } \\
\hline Fusion & $3(8.8)$ & $7(25.0)$ & $2(11.8)$ & NS & NS \\
\hline $\mathrm{PAD}$ & $8(23.5)$ & $8(28.6)$ & $3(17.6)$ & NS & NS \\
\hline Age (yr) & $11.82 \pm 1.48$ & $11.17 \pm 1.20$ & $11.76 \pm 1.50$ & NS & NS \\
\hline \multicolumn{6}{|l|}{ Atlas dimension (mm) } \\
\hline Dorsal arch height & $8.49 \pm 1.59$ & $7.70 \pm 2.03$ & $8.09 \pm 1.59$ & NS & NS \\
\hline PNAH & $4.02 \pm 0.68$ & $3.90 \pm 0.92$ & $4.19 \pm 0.93$ & NS & NS \\
\hline A-P dimension & $45.11 \pm 2.91$ & $44.19 \pm 3.28$ & $43.47 \pm 3.26$ & NS & NS \\
\hline \multicolumn{6}{|l|}{ Cranial base angle $\left({ }^{\circ}\right)$} \\
\hline n-s-ba & $134.24 \pm 4.96$ & $131.74 \pm 5.25$ & $130.57 \pm 4.20$ & $<0.05^{*}$ & NS \\
\hline \multicolumn{6}{|l|}{ Sagittal dimensions $\left({ }^{\circ}\right)$} \\
\hline ss-n-pg & $6.07 \pm 1.67$ & $5.14 \pm 1.78$ & $4.14 \pm 1.94$ & $<0.01^{* *}$ & NS \\
\hline s-n-ss & $80.33 \pm 2.89$ & $80.81 \pm 3.17$ & $80.95 \pm 3.01$ & NS & NS \\
\hline s-n-pg & $74.27 \pm 2.66$ & $75.69 \pm 3.79$ & $76.82 \pm 3.42$ & $<0.05^{*}$ & NS \\
\hline \multicolumn{6}{|l|}{ Vertical dimensions $\left({ }^{\circ}\right)$} \\
\hline $\mathrm{NL} / \mathrm{ML}$ & $27.88 \pm 4.04$ & $24.37 \pm 5.09$ & $27.57 \pm 7.73$ & NS & $<0.05^{*}$ \\
\hline NSL/NL & $8.73 \pm 3.17$ & $8.48 \pm 3.78$ & $6.74 \pm 2.11$ & NS & NS \\
\hline NSL/ML & $36.61 \pm 4.25$ & $32.85 \pm 6.77$ & $34.31 \pm 8.07$ & NS & NS \\
\hline \multicolumn{6}{|l|}{ Mandibular form $\left({ }^{\circ}\right)$} \\
\hline ML/Rlar & $121.34 \pm 5.54$ & $120.33 \pm 8.15$ & $124.47 \pm 6.99$ & NS & $<0.05^{*}$ \\
\hline ML/MBLar & $19.26 \pm 2.83$ & $19.95 \pm 2.96$ & $19.17 \pm 3.21$ & NS & NS \\
\hline \multicolumn{6}{|l|}{ Dental } \\
\hline Overjet (mm) & $8.64 \pm 2.11$ & $8.45 \pm 2.29$ & $7.25 \pm 2.29$ & $<0.001^{* * *}$ & $<0.01^{* *}$ \\
\hline Overbite (mm) & $2.91 \pm 1.88$ & $4.04 \pm 1.68$ & $1.41 \pm 3.35$ & NS & $<0.01$ \\
\hline ILs to NL $\left({ }^{\circ}\right)$ & $117.26 \pm 6.03$ & $117.41 \pm 6.80$ & $113.49 \pm 5.77$ & NS & NS \\
\hline ILi to ML $\left(^{\circ}\right)$ & $94.78 \pm 6.42$ & $98.69 \pm 6.67$ & $94.78 \pm 5.89$ & NS & NS \\
\hline Lower anterior facial height (sp-gn, mm) & $65.52 \pm 3.57$ & $60.02 \pm 4.67$ & $62.28 \pm 7.69$ & NS & $<0.05^{*}$ \\
\hline
\end{tabular}

Values are presented as number (\%) or mean \pm standard deviation.

Chi-square test for evaluation of CVM and upper spine morphology.

Multiple linear regression analysis for craniofacial morphology, adjusted for age, sex, and ethnicity.

$\mathrm{Z}$ vs. C, Significant difference between Z-activator and control; E vs. C, significant difference between E-activator and control; T1, before treatment; CVM, cervical vertebral maturation; CS1, cervical stage 1; CS2, cervical stage 2; CS3, cervical stage 3; CS4, cervical stage 4; PAD, posterior arch deficiency; PNAH, posterior neural arch height; A-P, anteroposterior; NS, not significant. ${ }^{*} p<0.05,{ }^{* *} p<0.01,{ }^{* * *} p<0.001$.

See Figures 2 and 3 for definitions of the other landmarks. 
Oh et al • Functional appliance-induced growth changes in Class Il malocclusion

Table 2. Comparison of true craniofacial changes and jaw rotations after Class II functional appliance treatment (T1-T2) with those in the absence of treatment (control group) (superimposition on stable structures)

\begin{tabular}{|c|c|c|c|c|c|}
\hline \multirow{2}{*}{ Variable } & \multirow{2}{*}{$\begin{array}{c}\mathrm{Z} \text {-activator } \\
(\mathrm{n}=\mathbf{3 4})\end{array}$} & \multirow{2}{*}{$\begin{array}{c}\text { E-activator } \\
(\mathbf{n}=\mathbf{2 8})\end{array}$} & \multirow{2}{*}{$\begin{array}{l}\text { Control } \\
(n=17)\end{array}$} & \multicolumn{2}{|c|}{$p$-value } \\
\hline & & & & Z vs. C & Evs. C \\
\hline Treatment duration (yr) & $1.55 \pm 0.67$ & $1.79 \pm 1.18$ & $1.46 \pm 0.51$ & NS & NS \\
\hline Age at T2 (yr) & $13.36 \pm 1.39$ & $12.89 \pm 1.45$ & $13.24 \pm 1.69$ & NS & NS \\
\hline \multicolumn{6}{|l|}{ Cranial base changes $\left(^{\circ}\right)$} \\
\hline n-s-ba & $-1.05 \pm 1.84$ & $-0.60 \pm 2.46$ & $0.38 \pm 1.61$ & $<0.001^{* * *}$ & $<0.05^{*}$ \\
\hline \multicolumn{6}{|l|}{ Sagittal changes $\left(^{\circ}\right)$} \\
\hline ss-n-pg & $-2.27 \pm 1.28$ & $-1.78 \pm 1.30$ & $-0.68 \pm 0.91$ & $<0.001^{* * *}$ & $<0.001^{* * *}$ \\
\hline s-n-ss & $-1.25 \pm 1.52$ & $-0.67 \pm 1.54$ & $0.17 \pm 0.81$ & $<0.001^{* * *}$ & $<0.05^{*}$ \\
\hline s-n-pg & $1.02 \pm 1.31$ & $1.10 \pm 1.56$ & $0.84 \pm 0.87$ & NS & NS \\
\hline \multicolumn{6}{|l|}{ Vertical changes $\left(^{\circ}\right)$} \\
\hline $\mathrm{NL} / \mathrm{ML}$ & $-1.59 \pm 1.72$ & $-1.10 \pm 1.78$ & $-0.94 \pm 2.47$ & NS & NS \\
\hline $\mathrm{NSL} / \mathrm{NL}$ & $0.82 \pm 1.36$ & $1.43 \pm 1.97$ & $0.94 \pm 1.85$ & NS & NS \\
\hline NSL/ML & $-0.79 \pm 1.65$ & $0.34 \pm 2.11$ & $-0.84 \pm 1.56$ & NS & NS \\
\hline \multicolumn{6}{|l|}{ Jaw rotations $\left({ }^{\circ}\right)$} \\
\hline NSL/Maxilla ${ }^{\text {Ref }}$ & $0.09 \pm 0.75$ & $0.44 \pm 1.53$ & $-0.43 \pm 1.01$ & NS & $<0.01^{* *}$ \\
\hline NSL/Mandible ${ }^{\text {Ref }}$ & $-1.30 \pm 1.94$ & $-1.02 \pm 2.42$ & $-1.67 \pm 2.20$ & NS & NS \\
\hline \multicolumn{6}{|l|}{ Condylar growth (mm) } \\
\hline cd-cd ${ }^{\text {Ref }}$ & $6.52 \pm 2.71$ & $8.62 \pm 3.47$ & $4.41 \pm 2.12$ & $<0.001^{* * *}$ & $<0.001^{* * *}$ \\
\hline $\operatorname{ar}-a^{\text {Ref }}$ & $6.52 \pm 2.71$ & $7.44 \pm 2.86$ & $4.41 \pm 2.20$ & $<0.01^{* *}$ & $<0.001^{* * *}$ \\
\hline \multicolumn{6}{|l|}{ Mandibular form changes $\left({ }^{\circ}\right)$} \\
\hline ML/Rlar & $0.21 \pm 2.20$ & $0.84 \pm 2.76$ & $-0.63 \pm 2.45$ & NS & NS \\
\hline ML/MBLar & $0.39 \pm 1.01$ & $-0.12 \pm 1.38$ & $0.24 \pm 1.42$ & NS & NS \\
\hline \multicolumn{6}{|l|}{ Upper teeth to Maxilla ${ }^{\text {Ref }}$} \\
\hline isi horizontal (mm) & $-2.54 \pm 2.20$ & $-1.75 \pm 1.83$ & $0.96 \pm 0.62$ & $<0.001^{* * *}$ & $<0.001^{* * *}$ \\
\hline isi vertical (mm) & $1.95 \pm 1.89$ & $2.69 \pm 2.16$ & $1.41 \pm 0.88$ & NS & $<0.05^{*}$ \\
\hline ILs inclination $\left(^{\circ}\right)$ & $-5.01 \pm 5.07$ & $-7.09 \pm 5.48$ & $1.89 \pm 2.86$ & $<0.001^{* * *}$ & $<0.001^{* * *}$ \\
\hline \multicolumn{6}{|l|}{ Lower teeth to Mandible ${ }^{\text {Ref }}$} \\
\hline iii horizontal (mm) & $0.53 \pm 1.59$ & $1.54 \pm 1.91$ & $0.29 \pm 1.15$ & NS & $<0.01^{* *}$ \\
\hline iii vertical (mm) & $1.66 \pm 1.30$ & $0.26 \pm 1.60$ & $0.87 \pm 1.45$ & $<0.05^{*}$ & NS \\
\hline ILi inclination $\left(^{\circ}\right)$ & $0.61 \pm 4.76$ & $4.47 \pm 6.96$ & $0.21 \pm 3.14$ & NS & $<0.001^{* * *}$ \\
\hline \multicolumn{6}{|l|}{ Dental relationship (mm) } \\
\hline Overjet & $-4.96 \pm 2.20$ & $-5.36 \pm 2.43$ & $0.32 \pm 1.01$ & $<0.001^{* * *}$ & $<0.001^{* * *}$ \\
\hline Overbite & $-0.65 \pm 1.60$ & $-2.03 \pm 2.02$ & $-0.11 \pm 1.67$ & NS & $<0.01^{* *}$ \\
\hline Lower anterior facial height (sp-gn, mm) & $3.22 \pm 1.84$ & $4.05 \pm 2.08$ & $1.83 \pm 1.65$ & $<0.05^{*}$ & $<0.001^{* * *}$ \\
\hline
\end{tabular}

Values are presented as mean \pm standard deviation.

Multiple linear regression analysis, adjusted for age, sex, treatment timing, treatment duration, and ethnicity.

$\mathrm{Z}$ vs. C, Significant difference between Z-activator and control; E vs. C, significant difference between E-activator and control; T1, before treatment; T2, after functional appliance treatment; $\operatorname{ar}_{-} \mathrm{ar}^{\mathrm{Ref}}$, articulare-articulare at T1; NS, not significant. ${ }^{*} p<0.05,{ }^{* *} p<0.01,{ }^{* * *} p<0.001$.

See Figures 2-5 definitions of the other landmarks. 
Table 3. Comparison of true craniofacial changes and jaw rotations after the retention period following Class II functional appliance treatment (T1-T3) with those in the absence of treatment (control group) (superimposition on stable structures)

\begin{tabular}{|c|c|c|c|c|c|}
\hline \multirow{2}{*}{ Variable } & \multirow{2}{*}{$\begin{array}{c}\text { Z-activator } \\
(\mathbf{n}=\mathbf{3 4})\end{array}$} & \multirow{2}{*}{$\begin{array}{c}\text { E-activator } \\
(\mathbf{n}=\mathbf{2 8})\end{array}$} & \multirow{2}{*}{$\begin{array}{l}\text { Control } \\
(\mathrm{n}=17)\end{array}$} & \multicolumn{2}{|c|}{$p$-value } \\
\hline & & & & Z vs. C & E vs. C \\
\hline Treatment duration (yr) & $3.58 \pm 1.25$ & $3.75 \pm 1.75$ & $3.41 \pm 0.97$ & NS & NS \\
\hline Age (yr) & $15.36 \pm 1.49$ & $14.84 \pm 1.90$ & $15.56 \pm 1.74$ & NS & NS \\
\hline \multicolumn{6}{|l|}{ Cranial base changes $\left({ }^{\circ}\right)$} \\
\hline n-s-ba & $-2.41 \pm 2.35$ & $-1.77 \pm 2.76$ & $-0.34 \pm 2.23$ & $<0.01^{* *}$ & NS \\
\hline \multicolumn{6}{|l|}{ Sagittal changes $\left({ }^{\circ}\right)$} \\
\hline ss-n-pg & $-2.19 \pm 1.53$ & $-2.17 \pm 1.44$ & $-0.92 \pm 1.33$ & $<0.001^{* * *}$ & $<0.01^{* *}$ \\
\hline s-n-ss & $-0.27 \pm 1.80$ & $-0.15 \pm 1.84$ & $0.56 \pm 1.13$ & $<0.01^{* *}$ & NS \\
\hline s-n-pg & $1.92 \pm 1.78$ & $2.00 \pm 1.76$ & $1.46 \pm 1.76$ & NS & NS \\
\hline \multicolumn{6}{|l|}{ Vertical changes $\left({ }^{\circ}\right)$} \\
\hline $\mathrm{NL} / \mathrm{ML}$ & $-1.23 \pm 2.60$ & $-1.45 \pm 2.19$ & $-1.72 \pm 2.84$ & NS & NS \\
\hline NSL/NL & $0.04 \pm 2.04$ & $0.96 \pm 1.82$ & $0.04 \pm 2.09$ & NS & NS \\
\hline NSL/ML & $-1.10 \pm 2.53$ & $-0.41 \pm 2.01$ & $-1.69 \pm 2.31$ & NS & NS \\
\hline \multicolumn{6}{|l|}{ Jaw rotations $\left({ }^{\circ}\right)$} \\
\hline NSL/Maxilla ${ }^{\text {Ref }}$ & $-0.86 \pm 1.03$ & $-0.32 \pm 1.66$ & $-0.79 \pm 1.28$ & NS & NS \\
\hline NSL/Mandible ${ }^{\text {Ref }}$ & $-2.34 \pm 3.41$ & $-2.49 \pm 2.68$ & $-3.19 \pm 3.08$ & NS & NS \\
\hline \multicolumn{6}{|l|}{ Condylar growth (mm) } \\
\hline$c d-$ cd $^{\text {Ref }}$ & $11.54 \pm 5.08$ & $12.74 \pm 5.16$ & $9.65 \pm 4.24$ & NS & NS \\
\hline ar-ar ${ }^{\text {Ref }}$ & $9.78 \pm 5.21$ & $10.35 \pm 4.90$ & $8.14 \pm 3.70$ & NS & NS \\
\hline \multicolumn{6}{|l|}{ Mandibular form changes $\left({ }^{\circ}\right)$} \\
\hline ML/Rlar & $-1.69 \pm 2.51$ & $-1.42 \pm 2.94$ & $-2.64 \pm 2.16$ & NS & NS \\
\hline ML/MBLar & $1.22 \pm 1.61$ & $0.77 \pm 1.29$ & $0.85 \pm 1.39$ & NS & NS \\
\hline \multicolumn{6}{|l|}{ Upper teeth to Maxilla $^{\text {Ref }}$} \\
\hline isi horizontal (mm) & $-2.28 \pm 3.39$ & $-1.53 \pm 2.87$ & $1.34 \pm 0.89$ & $<0.001^{* * *}$ & $<0.001^{* * *}$ \\
\hline isi vertical (mm) & $3.92 \pm 1.94$ & $4.39 \pm 2.45$ & $2.85 \pm 1.54$ & $<0.001^{* * *}$ & NS \\
\hline ILs inclination $\left({ }^{\circ}\right)$ & $-5.18 \pm 7.09$ & $-6.61 \pm 8.22$ & $2.21 \pm 2.37$ & $<0.001^{* * *}$ & $<0.001^{* * *}$ \\
\hline \multicolumn{6}{|l|}{ Lower teeth to Mandible ${ }^{\text {Ref }}$} \\
\hline iii horizontal (mm) & $1.24 \pm 2.36$ & $1.75 \pm 2.20$ & $0.43 \pm 1.21$ & NS & $<0.01^{* *}$ \\
\hline iii vertical (mm) & $1.96 \pm 2.21$ & $1.11 \pm 1.87$ & $2.00 \pm 1.94$ & NS & $<0.01^{* *}$ \\
\hline ILi inclination $\left({ }^{\circ}\right)$ & $4.14 \pm 7.69$ & $4.25 \pm 5.81$ & $0.83 \pm 3.47$ & NS & $<0.01^{* *}$ \\
\hline \multicolumn{6}{|l|}{ Dental relationship (mm) } \\
\hline Overjet & $-5.84 \pm 2.55$ & $-5.54 \pm 2.57$ & $0.48 \pm 1.25$ & $<0.001^{* * *}$ & $<0.001^{* * *}$ \\
\hline Overbite & $-1.50 \pm 1.38$ & $-1.96 \pm 1.49$ & $0.15 \pm 1.17$ & $<0.05^{*}$ & $<0.001^{* * *}$ \\
\hline Lower anterior facial height (sp-gn, mm) & $6.02 \pm 2.31$ & $6.51 \pm 3.17$ & $3.82 \pm 2.62$ & $<0.05^{*}$ & $<0.05^{*}$ \\
\hline
\end{tabular}

Values are presented as mean \pm standard deviation.

Multiple linear regression analysis, adjusted for age, sex, treatment timing, treatment duration, and ethnicity.

Z vs. C, Significant difference between Z-activator and control; E vs. C, significant difference between E-activator and control; T1, before treatment; T3, after retention; $\operatorname{ar}_{-} \operatorname{ar}^{\mathrm{Ref}}$, articulare-articulare at T1; NS, not significant.

${ }^{*} p<0.05,{ }^{* *} p<0.01,{ }^{* * *} p<0.001$.

See Figures 2-5 definitions of the other landmarks. 


\section{Reliability and method errors}

The reliability of the craniofacial and upper spinal morphology measurements and CVM method was evaluated by repeat measurement of 25 randomly selected sets after 1 month, as previously reported. ${ }^{17,18}$ The craniofacial morphology and CVM stages were analyzed by one author (E0), who also analyzed the upper spine morphology together with another author (LS). For the craniofacial analysis, the method errors according to Dahlberg $^{19}$ ranged from 0.14 to 2.12 , and the reliability coefficients according to Houston ${ }^{20}$ were 0.76 to 0.99 . The reliability of the CVM method $(\kappa=0.91)$ and upper spine morphology measurements $(\kappa=0.82)$ was excellent. ${ }^{17,18}$

\section{Statistical analysis}

The Shapiro-Wilk W test showed that all variables were normally distributed. The baseline craniofacial morphology and changes from $\mathrm{T} 1$ to $\mathrm{T} 2$ and $\mathrm{T} 1$ to $\mathrm{T} 3 \mathrm{in}$ the Z-activator and E-activator groups were compared with those in the control group using one-way analysis of variance in the initial step. Subsequently, significant variables were compared between the treatment and control groups using multiple linear regression analysis, with adjustment for age, sex, and ethnicity for the craniofacial morphology and additional adjustment for the treatment timing and duration for the $\mathrm{T} 1-\mathrm{T} 2$ and $\mathrm{T} 1-$ $\mathrm{T} 3$ changes. No comparisons were made between the two treatment groups in this study.

\section{RESULTS}

\section{Craniofacial and upper spine morphology at baseline}

There were no significant between-group differences in the upper spine morphology at baseline (Table 1). Compared with the control group, the Z-activator group exhibited a larger cranial base angle $(p<0.05)$, sagittal jaw relationship $(p<0.01)$, and overjet $(p<0.001)$ and a more retracted chin position $(p<0.05)$, while the E-activator group exhibited a smaller mandibular inclination $(p<0.05)$, gonial angle $(p<0.05)$, lower anterior facial height $(p<0.05)$, a larger overjet $(p<0.01)$ and overbite $(p<0.01$; Table 1$)$.

\section{Effects of functional appliance treatment from $\mathrm{T} 1$ to T2}

Relative to the findings in the control group, the cranial base angle $(p<0.001)$, sagittal jaw relationship ( $p$ $<0.001)$, maxillary prognathism $(p<0.001)$, and overjet $(p<0.001)$ were significantly decreased while condylar growth $(p<0.001)$ and the lower anterior facial height $(p<0.05)$ were significantly increased in the Z-activator group. Moreover, the maxillary incisors were significantly retroclined $(p<0.001)$ while the mandibular incisors were significantly extruded $(p<0.05)$ in the Z-activator group (Table 2).

In the E-activator group, the cranial base angle $(p<$ $0.05)$, sagittal jaw relationship ( $p<0.001)$, maxillary prognathism $(p<0.05)$, overjet $(p<0.001)$, and overbite $(p<0.01)$ were significantly decreased while condylar growth $(p<0.001)$, rotation of the maxilla $(p<0.01)$, and the lower anterior facial height $(p<0.001)$ were significantly increased relative to the changes in the control group. Moreover, the maxillary incisors were significantly retroclined $(p<0.001)$ and extruded $(p<0.05)$ while the mandibular incisors were significantly proclined $(p<$ 0.001 ) relative to those in the control group (Table 2).

\section{Effects of functional appliance treatment from $\mathrm{T} 1$ to T3}

Compared with the control group, the Z-activator group exhibited significant decreases in the cranial base angle $(p<0.01)$, sagittal jaw relationship $(p<0.001)$, maxillary prognathism $(p<0.01)$, overjet $(p<0.001)$, and overbite $(p<0.05)$ and a significant increase in the lower anterior facial height $(p<0.05)$ after retention. Moreover, the maxillary incisors were significantly retroclined $(p<0.001)$ and extruded $(p<0.001)$ relative to those in the untreated control group (Table 3).

In the E-activator group, the sagittal jaw relationship $(p<0.01)$, overjet $(p<0.001)$, and overbite $(p<0.001)$ were significantly decreased while the lower anterior facial height $(p<0.05)$ was significantly increased after retention. The maxillary incisors were significantly retroclined $(p<0.001)$ and the mandibular incisors were significantly proclined $(p<0.01)$ and intruded $(p<0.01)$ relative to those in the control group (Table 3).

\section{DISCUSSION}

In the present study, true dentoalveolar, skeletal, and rotational changes induced by functional appliances were investigated using Björk's structural method of superimposition in growing children with Class 11 malocclusion, and the findings were compared with the growth changes in untreated matched controls. ${ }^{7-9}$ To the best of our knowledge, this is the first study to analyze true dentoalveolar, skeletal, and rotational changes induced by Class 11 functional appliances by using the structural method.

The control group included historical cephalograms retrieved from the archives of Björk's classic implant studies. ${ }^{7-9}$ Previously, it has been reported that the use of historical control samples can deflate the treatment effect of functional appliances because of a secular change in the growth pattern. ${ }^{21}$ Thus, the treatment effects found in the present study may be conservatively interpreted. 


\section{Craniofacial and upper spine morphology at baseline}

At baseline, the mandible was significantly more retracted in the Z-activator group than in the control group, while the mandibular plane angle in the E-activator group was lower than that in the control group. These differences can be attributed to the indications of the two appliances. ${ }^{10,11}$ Because both appliances have different indications, and the children in the two treatment groups were not randomly distributed at baseline, the Z-activator and E-activator groups were only compared with the control group and not with each other. $^{10,11}$

The upper spine morphology and atlas dimensions at baseline were comparable between groups. This was expected because the patients in all three groups had skeletal Class 11 Division 1 malocclusion. ${ }^{17,22-24}$

\section{Short-term effects of the functional appliances (T1-T2)}

Both the Z-activator and the E-activator were effective in improving the sagittal jaw and dentoalveolar relationships in the short term. The improvement in the sagittal jaw relationship was achieved by restricted maxillary growth and additional condylar growth, although the significant condylar growth was not associated with significant advancement of the chin. It is generally agreed that functional appliances can accelerate mandibular growth on a short-term basis, although there is controversy regarding sagittal advancement of the chin position ${ }^{1-5}$ due to the association between the sagittal and vertical dimensions. ${ }^{7-9}$

Teuscher ${ }^{10}$ previously pointed out that posterior rotation of the maxilla is the major component that forces the mandible to rotate posteriorly, and he proposed the Z-activator, a combined headgear-activator appliance, for prevention of this unfavorable effect. In the present study, significant backward rotation of the maxilla was observed in the E-activator group, whereas this change was insignificant in the Z-activator group. Backward rotation of the maxilla and vertical development of the maxillary alveolar structures may counteract additional condylar growth; therefore, advancement of the chin position was not evident in the present study. ${ }^{10}$

Previous studies have reported backward rotation of the mandible after functional appliance treatment; ${ }^{4,5,10,11}$ however, mandibular rotation was not evident in the present study. Instead, the cranial base angle exhibited a decrease in the treated groups. The cranial base may have played a role in counteraction of backward rotation of the mandible in the present study. A compensating mechanism associated with the cranial base angle has previously been described, and it was found that a more flexed cranial base angle was associated with forward rotation of the mandible. ${ }^{25,26}$ In particular, the posterior part of the cranial base may be the area of interest in growth modification treatment, because cellular activity in the spheno-occipital synchondrosis and the remodeling process are reportedly observed until late puberty. ${ }^{25,26}$

\section{Long-term treatment effects of the functional appliances (T1-T3)}

Some authors argue that the growth increase induced by functional appliances is temporary, and that the effects disappear by the time of growth completion such that the outcomes are similar to the expected growth in the absence of treatment. ${ }^{3-5}$ In the present study, the treated children presented significant condylar growth acceleration after active treatment, which became insignificant during retention at the postpubertal growth peak. The treated groups maintained an additional condylar growth of $1.5-3 \mathrm{~mm}$, similar to the findings in previous studies. ${ }^{1,2}$ However, the growth exhibited large individual variations and failed to show statistical significance on a long-term basis, even though the true changes were analyzed by superimposition on the stable structures. The results suggest the importance of case selection for functional appliance treatment and highlight the need to focus more on the phenotype of the skeletal class 11 malocclusion for optimal treatment success.

Prediction of jaw growth and rotation patterns is crucial for successful treatment with Class II functional appliances. ${ }^{27}$ Recent studies found that the upper spine morphology and atlas dimensions were significantly associated with growth prediction signs, and it was recommended that the upper spinal morphology and atlas dimensions should be considered during orthodontic treatment planning for growing children. ${ }^{7-9,17,22}$ Therefore, future studies should investigate the association between the upper spinal morphology and the success of treatment with Class 11 functional appliances.

We observed significant long-term improvement in the skeletal jaw relationship after Class 11 functional appliance treatment. The long-term effect on the maxilla was in agreement with that observed in previous studies, although the effect on the mandible is still a debatable topic. ${ }^{1-5,10,11}$ Some authors suggest that functional appliance treatment during the pubertal growth spurt can produce more skeletal effects, such as significant advancement of the chin.' However, in the present study, advancement of the chin position was not significant even after adjustment for the possible effect of the treatment timing (before or during the growth peak).

Both treated groups in the present study exhibited a significant long-term improvement in the dental relationship, consistent with the findings in previous studies. ${ }^{1-4,10,11}$ However, proclination of the mandibular incisors was only observed in the E-activator group and was not significant in the Z-activator group. It has been re- 
ported that inclination of the mandibular incisors is less affected by an activator-headgear combination than by an activator alone..$^{28}$ In the present study, changes in the mandibular incisors were analyzed by superimposition on the stable structures, which is independent from the remodeling of the lower mandibular surface. ${ }^{7-9}$ The findings suggest that a Z-activator is indicated when labial tipping of the mandibular incisors is not desirable.

\section{CONCLUSION}

In the short term, both Z- and E-activator were effective in improving sagittal jaw relationships, dental relationship and accelerating condylar growth. In the long term, the sagittal jaw relationship and the dental relationship were significantly improved, but additional condylar growth, vertical and rotational changes in the jaws were not significant.

\section{CONFLICTS OF INTEREST}

No potential conflict of interest relevant to this article was reported.

\section{ACKNOWLEDGEMENTS}

We thank Jens Bjørn Jørgensen for technical support in the TIOPS analysis.

\section{REFERENCES}

1. Cozza P, Baccetti T, Franchi L, De Toffol L, McNamara JA Jr. Mandibular changes produced by functional appliances in Class 11 malocclusion: a systematic review. Am J Orthod Dentofacial Orthop 2006;129:599.e1-12; discussion e1-6.

2. Zelderloo A, Cadenas de Llano-Pérula M, Verdonck A, Fieuws S, Willems G. Cephalometric appraisal of Class 11 treatment effects after functional and fixed appliances: a retrospective study. Eur J Orthod 2017;39:334-41.

3. Batista KB, Thiruvenkatachari B, Harrison JE, O'Brien KD. Orthodontic treatment for prominent upper front teeth (Class 11 malocclusion) in children and adolescents. Cochrane Database Syst Rev 2018;3:CD003452.

4. Tulloch JF, Proffit WR, Phillips C. Outcomes in a 2-phase randomized clinical trial of early Class 11 treatment. Am J Orthod Dentofacial Orthop 2004; 125:657-67.

5. Cross JJ. Facial growth: before, during, and following orthodontic treatment. Am J Orthod 1977;71: 68-78.

6. Arat ZM, Türkkahraman H, English JD, Gallerano RL,
Boley JC. Longitudinal growth changes of the cranial base from puberty to adulthood. A comparison of different superimposition methods. Angle Orthod 2010;80:537-44.

7. Björk A. Prediction of mandibular growth rotation. Am J Orthod 1969;55:585-99.

8. Björk A, Skieller V. Facial development and tooth eruption. An implant study at the age of puberty. Am J Orthod 1972;62:339-83.

9. Björk A, Skieller V. Normal and abnormal growth of the mandible. A synthesis of longitudinal cephalometric implant studies over a period of 25 years. Eur J Orthod 1983;5:1-46.

10. Teuscher U. A growth-related concept for skeletal class 11 treatment. Am J Orthod 1978;74:258-75.

11. Andresen V. The Norwegian system of functional gnatho-orthopedics. Acta Gnathol 1936;1:5-36.

12. Baccetti T, Franchi L, McNamara Jr JA. The cervical vertebral maturation (CVM) method for the assessment of optimal treatment timing in dentofacial orthopedics. Semin Orthod 2005;11:119-29.

13. Siersbæk-Nielsen S, Solow B. Intra- and interexaminer variability in head posture recorded by dental auxiliaries. Am J Orthod 1982;82:50-7.

14. Solow B, Tallgren A. Head posture and craniofacial morphology. Am J Phys Anthropol 1976;44:417-35.

15. Sandham A. Cervical vertebral anomalies in cleft lip and palate. Cleft Palate J 1986;23:206-14.

16. Huggare J. Association between morphology of the first cervical vertebra, head posture, and craniofacial structures. Eur J Orthod 1991;13:435-40.

17. Oh E, Ahn SJ, Sonnesen L. Ethnic differences in craniofacial and upper spine morphology in children with skeletal Class 11 malocclusion. Angle Orthod 2018;88:283-91.

18. Sonnesen L, Kjaer 1. Cervical vertebral body fusions in patients with skeletal deep bite. Eur J Orthod 2007;29:464-70.

19. Dahlberg G. Statistical methods for medical and biological students. New York: Interscience; 1940. p. 122-32.

20. Houston WJ. The analysis of errors in orthodontic measurements. Am J Orthod 1983;83:382-90.

21. Papageorgiou SN, Koretsi V, Jäger A. Bias from historical control groups used in orthodontic research: a meta-epidemiological study. Eur J Orthod 2017; 39:98-105.

22. Oh E, Ahn SJ, Sonnesen L. Ethnic differences in craniofacial and upper spine morphology between European and Asian children with skeletal Class 111 malocclusion. Am J Orthod Dentofacial Orthop 2019;156:502-11.

23. Arntsen T, Sonnesen L. Cervical vertebral column morphology related to craniofacial morphology and 
head posture in preorthodontic children with Class 11 malocclusion and horizontal maxillary overjet. Am J Orthod Dentofacial Orthop 2011;140:e1-7.

24. Sonnesen L, Kjaer 1. Anomalies of the cervical vertebrae in patients with skeletal Class 11 malocclusion and horizontal maxillary overjet. Am J Orthod Dentofacial Orthop 2008;133:188.e15-20.

25. Melsen B. Time of closure of the spheno-occipital synchondrosis determined on dry skulls. A radiographic craniometric study. Acta Odontol Scand 1969;27:73-90.

26. Solow B. The dentoalveolar compensatory mecha- nism: background and clinical implications. Br J Orthod 1980;7:145-61.

27. Petrovic AG, Stutzmann JJ. The concept of mandibular tissue-level growth potential and the responsiveness to a functional appliance. In: Graber LW, Graber TM, eds. Orthodontics, state of the art, essence of the science. St. Louis: Mosby Co; 1986. p. 59-74.

28. Türkkahraman H, Sayin MO. Effects of activator and activator headgear treatment: comparison with untreated Class 11 subjects. Eur J Orthod 2016;28:2734. 